\title{
Improvement of Air Fuel Ratio Model Using a Least Absolute Shrinkage and Selection Operator
}

\author{
Chanyut Khajorntraidet* \\ Graduate School of Engineering and Science \\ Shibaura Institute of Technology \\ Saita ma 337-8570, Japan \\ *Corresponding author
}

\author{
Tielong Shen \\ Department of Engineering and Applied Sciences \\ Sophia University \\ Tokyo 102-8554, Japan
}

\begin{abstract}
-the improvement of an air-fuel ratio model based on in-cylinder pressure measurement is presented. This model has been applied for calculation of static air-fuel ratio of spark ignition (SI) engines. In addition, it can be replaced the lambda sensors in feedback air-fuel ratio control systems. However the parameters of the model obtained from ridge regression method compose of few small coefficients, in practical applications, these small coefficients cannot be ignored because they affect the airfuel ratio calculation error. Therefore, the authors propose modification of the air-fuel ratio model structure and also the model identification method using a least absolute shrinkage and selection operator (LASSO). With the application of this method, some coefficients are shrunk to zero then the model structure is simplified. The results compared with ridge regression show that this proposed method can improve the structure of the air-fuel ratio model without increasing of the calculation error.
\end{abstract}

Keywords-spark ignition engine; in-cylinder pressure; air-fuel ratio estimation; least absolute sh rinkage and selection operator

\section{INTRODUCTION}

The possibility of sensing the air-fuel ratio of spark ignition (SI) engines leads to the development of effective air-fuel ratio control strategies. There are many researches that have been presented about in-cylinder pressure applications for internal combustion engines control and analysis [1-3]. For the air-fuel ratio estimation based on in-cylinder pressure measurement, there are many approaches that have been applied, for example [4].In previous work [5], we have been introduced the air-fuel ratio model based on in-cylinder pressure measurement for calculation of the static air-fuel ratio of the SI engine. The model requires engine speed, intake manifold pressure, including rapid burn angle and heat release as model inputs, while the model identification, a ridge regression has been applied. The implementation of this method can overcome the singular problem caused by an experimental data set. The ridge regression method is same as ordinary least squares except for shrinking the estimated coefficients towards zero and it also makes the problem nonsingular. This was the main motivation for the ridge regression when it was first introduced in statistics [6].The design of ridge coefficient can minimize a penalized

\author{
Kazuhisa Ito \\ Department of Machinery and Control Systems \\ College of Systems Engineering and Science \\ Shibaura Institute of Technology \\ Saita ma 337-8570, Japan
}

residual sum of squares. However, the ridge regression has some drawbacks such as small calculated model coefficients in the model structure. We cannot ignore effects of these small coefficient values in the model because this will lead to air-fuel ratio calculation error. Furthermore, the ridge regression technique generally affects the squared bias compared with the ordinary least squares.

Hence, we propose the improvement of the air-fuel ratio model identification by applying a least absolute shrinkage and selection operator (LASSO) method which was proposed by R. Tibshirani [7], this technique has more efficiency than the ordinary least squares and the ridge regression. It can shrink some coefficients and set other to zero, also retain the good features of both subset selection and ridge regression [8].W ith this identification technique, the results show that the model structure is simplified because some coefficients are shrunk to zero. Moreover, the sum of square error of model is not affected by this technique compared with the case of using ridge regression.

This paper organized as follows: first, the relationship between in-cylinder pressure and air-fuel ratio is introduced and the polynomial approximation model of air-fuel ratio is presented. The system identification processes using ridge regression and LASSO are then expressed. Subsequently, the identification results for the model comparis on are investigated. Finally, all research results are discussed.

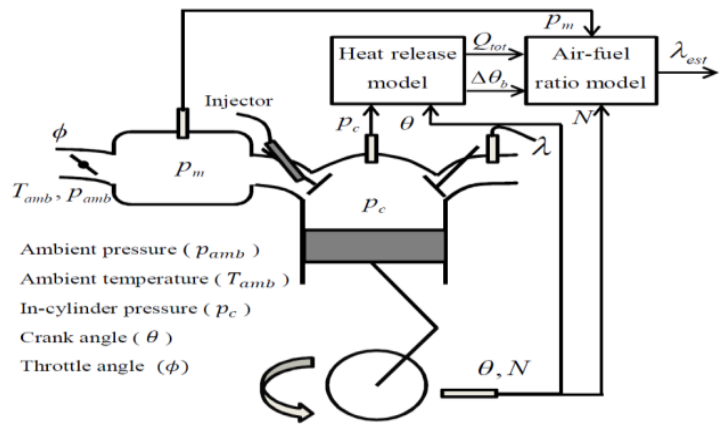

FIGURE I. AIR-FUEL RATIO ESTIMATION MODEL 


\section{AIR FUEL RATIO MODEL}

The notion of the air-fuel ratio calculation and some required inputs are shown in Fig. 1. The cylinder pressurebased air-fuel ratio model inputs are the rapid burn angle, heat release, intake manifold pressure, and the engine speed. The dynamic behavior of each input is regarded as the selection of the input order in the model structure. The intake manifold pressure and engine speed have a strong effect on the air mass flow rate entering the combustion chamber. Additionally, the values of the heat release $\left(Q_{\text {tot }}\right)$ and the rapid burn angle $\left(\Delta \theta_{b}\right)$ are depending on the fuel mass injected into the cylinder. Therefore, the quantity of all inputs can be used to estimate the air-fuel ratio of SI engines.

The total heat release for the air-fuel ratio model is obtained fro $m$ the integration of the heat release rate fro $m$ the start to the end of combustion [9]. In this work, the effects of crevice volumes and heat transfer are ignored. Hence, the total heat release denotes the total energy which calculates from the incylinder pressure measurement during combustion period. The air-fuel ratio model is introduced with the combination of the Taylor series expansion as follows:

$$
\begin{aligned}
\lambda=\lambda_{0} & +\alpha_{1}\left[\left(\Delta \theta_{b}-\Delta \theta_{b 0}\right) / N\right]+\alpha_{2}\left(Q_{t o t}-Q_{t o t, 0}\right) \\
& +\alpha_{3}\left[\left(\Delta \theta_{b}-\Delta \theta_{b, 0}\right) / N\right]^{2}+\alpha_{4}\left[\left(\Delta \theta_{b}-\Delta \theta_{b, 0}\right) / N\right]\left(Q_{t o t}-Q_{t o t, 0}\right) \\
& +\alpha_{5}\left(Q_{t o t}-Q_{t o t, 0}\right)^{2}+\alpha_{6}\left(p_{m}-p_{m, 0}\right)+\alpha_{7}\left(N-N_{0}\right)
\end{aligned}
$$

where $\lambda$ is air-fuel ratio, $\lambda_{0}$ is the stoichiometric air-fuel ratio, i.e., $\lambda_{0}=\mathrm{f}\left(\mathrm{p}_{\mathrm{m}, 0}, \mathrm{~N}_{0}, \Delta \theta_{\mathrm{b}, 0}, \mathrm{Q}_{\mathrm{tot}, 0}\right)$. The subscript zero of each input, refer to the initial value at stoichiometric air-fuel ratio. For the model inputs, $\mathrm{p}_{\mathrm{m}}$ is the intake manifold pressure $(\mathrm{Pa})$, $\mathrm{N}$ is the engine speed (rpm), $\mathrm{Q}_{\text {tot }}$ is the total heat release $(\mathrm{kJ})$ and $\Delta \theta_{\mathrm{b}}$ is the rapid burn angle (degrees). This equation utilizes only linear terms of the intake manifold pressure and engine speed because their dynamic behaviors are slower than the combustion parameters obtained from in-cylinder pressure data. In the next section, the identification process for the airfuel ratio model is expressed.

\section{SYSTEM IDENTIFICATION}

In this identification, considering a steady state air-fuel ratio response, both the intake manifold pressure and the engine speed have a significant effect on the air mass flow rate. The fuel mass directly affects the total heat release and burn duration. The main air-fuel ratio oscillation is caused by the total heat release and the rapid burn angle because the changing rate of the manifold pressure and the engine speed are slower compared with the variation of the combustion parameters. The air-fuel ratio and four input values under various operating conditions are obtained from experiments by averaging over 500 working cycles. During the torque constant mode experiments, both changing of fuel injection and engine speed are considered. According to the experimental conditions, the changing of the air-fuel ratio is assumed to be caused only by the fuel injection command. The air mass flow rate going through the combustion chamber is almost constant because the throttle angle is fixed. The polynomial parameter estimation for the model is created as follows:

$$
\begin{aligned}
& \lambda-\lambda_{0}=\left[\begin{array}{lll}
\left.\left(\Delta \theta_{b}-\Delta \theta_{b 0}\right) / N\right) & \left(Q_{t o t}-Q_{t o t, 0}\right) & \left(\left(\Delta \theta_{b}-\Delta \theta_{b, 0}\right) / N\right)^{2}
\end{array}\right. \\
& \left(\left(\Delta \theta_{b}-\Delta \theta_{b, 0}\right) / N\right)\left(Q_{t o t}-Q_{t o t, 0}\right) \quad\left(Q_{t o t}-Q_{t o t, 0}\right)^{2} \quad\left(p_{m}-p_{m, 0}\right) \\
& \left.\left(N-N_{0}\right)\right] \cdot\left[\begin{array}{lllllll}
\alpha_{1} & \alpha_{2} & \alpha_{3} & \alpha_{4} & \alpha_{5} & \alpha_{6} & \alpha_{7}
\end{array}\right]^{T}
\end{aligned}
$$

The regression vector is

$$
\begin{aligned}
& \varphi=\left[\left[\begin{array}{ll}
\left.\left(\Delta \theta_{b}-\Delta \theta_{b 0}\right) / N\right] & \left(Q_{t o t}-Q_{t o t, 0}\right)
\end{array}\right.\right. \\
& {\left[\left(\Delta \theta_{b}-\Delta \theta_{b, 0}\right) / N\right]^{2}\left[\left(\Delta \theta_{b}-\Delta \theta_{b, 0}\right) / N\right]\left(Q_{t o t}-Q_{t o t, 0}\right)} \\
& \left.\left(Q_{t o t}-Q_{t o t, 0}\right)^{2} \quad\left(p_{m}-p_{m, 0}\right) \quad\left(N-N_{0}\right)\right]
\end{aligned}
$$

Define the parameter vector as

$$
\alpha=\left[\begin{array}{lllllll}
\alpha_{1} & \alpha_{2} & \alpha_{3} & \alpha_{4} & \alpha_{5} & \alpha_{6} & \alpha_{7}
\end{array}\right]^{T}
$$

Hence, the model output become

$$
y=\lambda-\lambda_{0}=f\left(p_{m}, N, \Delta \theta_{b}, Q_{t o t}\right)-f\left(p_{m, 0}, N_{0}, \Delta \theta_{b, 0}, Q_{t o t, 0}\right)
$$

From (5), the outputsy from measurement are collected in to an output vector $Y$ and the regression vectors $\varphi$ are collected into a regression matrix $\Phi$.

$$
Y=\left[\begin{array}{lllll}
y_{1} & y_{2} & y_{3} & \cdots & y_{n}
\end{array}\right]^{T} \text { and } \Phi=\left[\begin{array}{llll}
\varphi_{1} & \varphi_{2} & \varphi_{3} & \cdots
\end{array} \varphi_{n}\right]^{T}
$$

The estimated of model coefficients can be calculated by many methods. In this work, the implement of ridge regression and LASSO will be presented in the next section.

\section{A. Ridge Regression}

Ridge regression shrinks the regression coefficients by imposing a penalty on their size. The ridge coefficients minimize a penalized residual sum of squares (RSS)

$$
\hat{\alpha}_{\text {ridge }}=\underset{\alpha}{\arg \min }\left\{\sum_{i=1}^{n}\left(y_{i}-\alpha_{0}-\sum_{j=1}^{p} x_{i j} \alpha_{j}\right)^{2}+k \sum_{j=1}^{p} \alpha_{j}^{2}\right\}
$$

where $\mathrm{k} \geq 0$ is a complexity parameter that controls the amount of shrinkage; the larger the value ofk, the greater the amount of shrinkage.n is the number of measurements, $y_{i}$ is the response at observation $\mathrm{i}, \mathrm{x}_{\mathrm{ij}}$ is data, at elementjof a regression vector at observation $\mathrm{i}, \alpha_{0}$ and $\alpha$ are scalar and $\mathrm{p}$ is the number of vector elements, respectively. $\alpha_{0}$ is estimated by $\bar{y}=\frac{1}{n} \sum_{1}^{n} y_{i}$. We can write the criterion (7) in matrix form

$$
R S S(k)=(Y-\Phi \alpha)^{T}(Y-\Phi \alpha)+k \alpha^{T} \alpha
$$


The ridge regression is applied to this problem for estimating the model coefficients because the determinants of $\Phi T \Phi$ is close to zero. The coefficients of the air-fuel ratio model can be calculated by using the following close form solution:

$$
\hat{\alpha}_{\text {ridge }}=\left(\Phi^{T} \Phi+k I\right)^{-1} \Phi^{T} Y
$$

where I is an identity matrix.

\section{B. LASSO}

LASSO is a regularization technique, which uses to solve the problem as follows:

$$
\hat{\alpha}_{\text {lasso }}=\underset{\alpha}{\arg \min }\left\{\sum_{i=1}^{n}\left(y_{i}-\alpha_{0}-\sum_{j=1}^{p} x_{i j} \alpha_{j}\right)^{2}+k \sum_{j=1}^{p}\left|\alpha_{j}\right|\right\}
$$

Notice that the similarity to the ridge regression problem (7) but the ridge $L_{2}$ penalty term $\mathrm{k} \sum_{\mathrm{j}=1}^{\mathrm{p}} \alpha_{\mathrm{j}}^{2}$ is replaced by the $\mathrm{LASSOL}_{1}$ penalty $\mathrm{k} \sum_{\mathrm{j}=1}^{\mathrm{p}}\left|\alpha_{\mathrm{j}}\right|$. This latter constraint makes the solutions nonlinear in the $y_{i}$, and there is no closed form expression as in ridge regression. Computing the lasso solution is a quadratic programming problem with the same computational cost as for ridge regression [8].

\section{IDENTIFICATION RESULTS}

Two cases of identification method are applied. We consider the structure of the air-fuel ratio model as introduced in the previous section. MATLAB program is applied to calculate the solutions of both techniques.

TABLE I. EXPERIMENTAL DATA FOR SYST EM IDENT IFICATION

\begin{tabular}{|c|c|c|c|c|c|}
\hline Condition No. & $\lambda-\lambda_{0}$ & $\mathrm{p}_{\mathrm{m}}(\mathrm{kPa})$ & $\mathrm{N}(\mathrm{rpm})$ & $\Delta \theta_{\mathrm{b}}(\mathrm{deg})$ & $\mathrm{Q}_{\text {tot }}(\mathrm{kJ})$ \\
\hline 1 & -0.7747 & 44.877 & 979.2 & 20.25 & 0.4466 \\
\hline 2 & -0.4498 & 44.841 & 978.5 & 21.34 & 0.4451 \\
\hline 3 & -0.0074 & 44.79 & 977.6 & 21.19 & 0.4484 \\
\hline 4 & 0.8568 & 45.659 & 954.2 & 20.37 & 0.4438 \\
\hline 5 & 2.0011 & 46.827 & 919.3 & 21.38 & 0.4463 \\
\hline 6 & -0.9221 & 44.026 & 1036.2 & 21.08 & 0.4514 \\
\hline 7 & -0.5057 & 44.135 & 1032.3 & 20.43 & 0.4423 \\
\hline 8 & 0.0000 & 44.397 & 1025.1 & 22.82 & 0.4469 \\
\hline 9 & 0.6279 & 44.899 & 1013.9 & 21.15 & 0.4442 \\
\hline 10 & 1.8810 & 46.429 & 977.8 & 21.60 & 0.4487 \\
\hline 11 & -1.0265 & 43.914 & 1084.7 & 21.59 & 0.4262 \\
\hline 12 & -0.6247 & 43.79 & 1085.9 & 21.78 & 0.4233 \\
\hline 13 & -0.2371 & 43.837 & 1081.7 & 21.95 & 0.4228 \\
\hline 14 & 0.4747 & 44.275 & 1065.2 & 22.06 & 0.4236 \\
\hline 15 & 1.6571 & 45.417 & 1034.4 & 22.65 & 0.4259 \\
\hline
\end{tabular}

\section{A. Identification Using Ridge Regression}

For ridge regression, the determinant of the matrix $\left(\Phi^{\mathrm{T}} \Phi+\right.$ $\mathrm{kI}$ ) is regulated by an adjustment of the complexity parameterk. This is because, the diagonal terms of considered matrix is depended on the value of $\mathrm{k}$. Therefore, we can solve the singular problem using the ridge regression. However, the value of parameter $\mathrm{k}$ affects the square bias of the estimation output. The experimental data for this identification is exhibited in Table 1. For this experiment, the center of an operating point is set at a condition No.8. The results of identification using the ridge regression method with $\mathrm{k}=0.06$ are shown in Table 2 .

\section{TABLE II. MODEL COEFFICIENT S CALCULATED BY RIDGE} REGRESSION

\begin{tabular}{|c|c|}
\hline Coefficient name & Value \\
\hline$\alpha_{1}$ & 0.1053 \\
\hline$\alpha_{2}$ & 0.3144 \\
\hline$\alpha_{3}$ & -0.0002 \\
\hline$\alpha_{4}$ & -0.0010 \\
\hline$\alpha_{5}$ & -0.0074 \\
\hline$\alpha_{6}$ & 1.2644 \\
\hline$\alpha_{7}$ & 0.0110 \\
\hline Intercept & -0.1967 \\
\hline
\end{tabular}

From the identification results, there are few model coefficients which are closed to zero. The intercept in this table represents $\alpha_{0}$ in (7). In practical applications, we cannot omit these small coefficients because they will lead to the increasing in estimation error.

\section{B. Identification Using Ridge LASSO}

This section presents the identification results using LASSO technique. First, the cross validation is applied for investigation the relation between the complexity parameterk and the mean square error (MSE) for this data set. The cross validation plot is shown in Fig. 2. According to these results, we can design the value of complexity parameter using one standard error rule for increasing regularization.

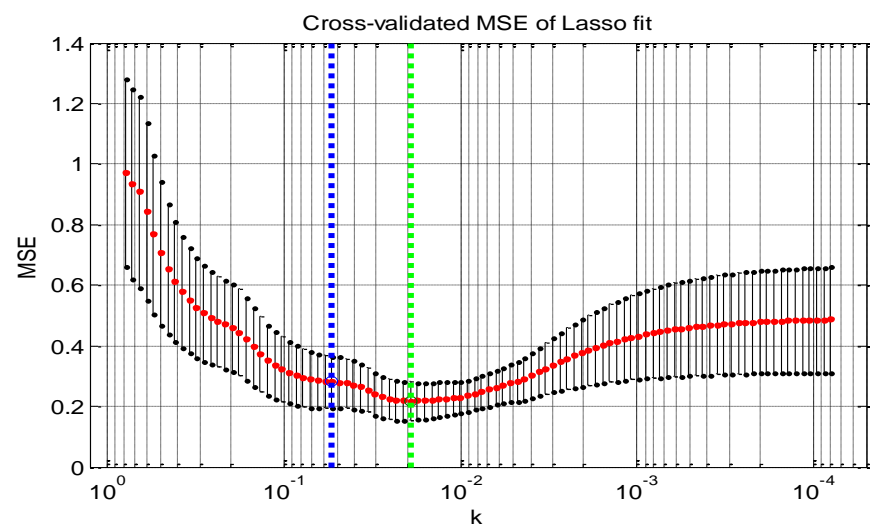

FIGURE II. CROSS VALIDATION PLOT

However, this parameter cannot minimize the MSE of estimation. This is caused by the number of non-zero coefficients, i.e., at the lowest MSE position, there are more non-zero coefficients than the prescribed period. Next, the relation between the complexity parameter and the number of non-zero coefficients is considered. The trace plot of scaled coefficients fit by LASSO is shown in Fig. 3. In this figure, the parameter df denotes the number of non-zero coefficients which depended on the complexity parameter that controls the amount of shrinkage. Finally, the calculation is performed using MATLAB program. The comple xity parameter is chosen as 0.06 and the estimated non-zero coefficients are five. Other coefficients are shrunk to zero. 


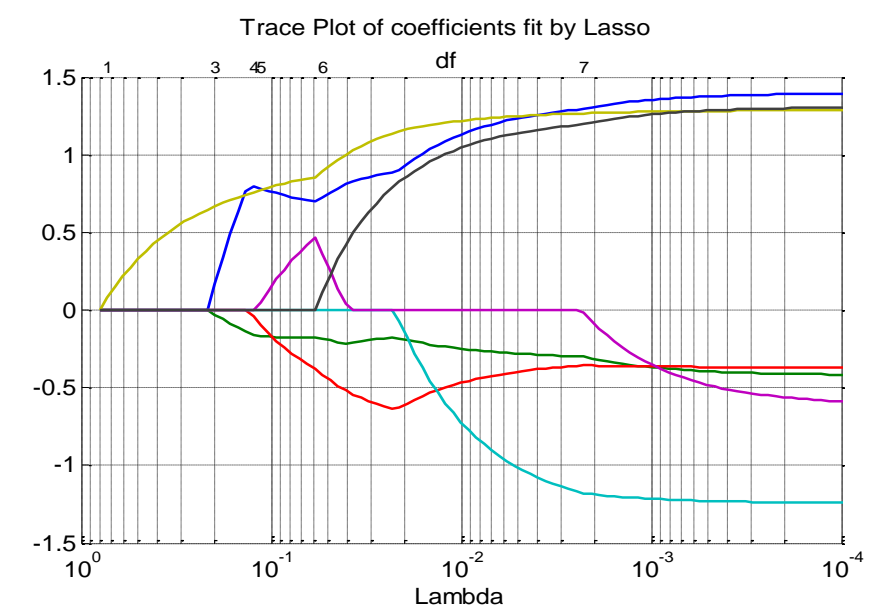

FIGURE III. TRACE PLOT OF MODEL COEFFICIENT S

Both the model coefficients multiplied with its scaling factor and the model intercept are exhibited in the Table 3.

TABLE III. MODEL COEFHCIENT S CALCULATED BY LASSO

\begin{tabular}{|c|c|}
\hline Coefficient name & Value \\
\hline$\alpha_{1}$ & 69.8238 \\
\hline$\alpha_{2}$ & -17.9928 \\
\hline$\alpha_{3}$ & -37198.5403 \\
\hline$\alpha_{4}$ & 0.0000 \\
\hline$\alpha_{5}$ & 454.0999 \\
\hline$\alpha_{6}$ & 0.8527 \\
\hline$\alpha_{7}$ & 0.0000 \\
\hline Intercept & -0.2216 \\
\hline
\end{tabular}

With the coefficients obtained by applying LASSO technique, the model is simplified and the structure is changed a little bit by adding an intercept term. Two terms of the original model are deducted and the values of remaining coefficients are changed. The intercept term is the value of $\alpha_{0}$ defined by (10). The model after simplified is as follows:

$$
\begin{aligned}
\lambda_{\text {est }}=( & \left.\lambda_{0}+\text { Intercept }\right)+\alpha_{1}\left[\left(\Delta \theta_{b}-\Delta \theta_{b 0}\right) / N\right] \\
& +\alpha_{2}\left(Q_{t o t}-Q_{t o t, 0}\right)+\alpha_{3}\left[\left(\Delta \theta_{b}-\Delta \theta_{b, 0}\right) / N\right]^{2} \\
& +\alpha_{5}\left(Q_{t o t}-Q_{t o t, 0}\right)^{2}+\alpha_{6}\left(p_{m}-p_{m, 0}\right)
\end{aligned}
$$

On the other hand, we can design the complexity parameter value for more shrinkage. This can be regarded based on cross validation and trace plot of coefficients. It will make the number of reduced non-zero coefficients but we have to consider about the increasing of MSE.

\section{MSE evaluation}

The air-fuel ratio model estimation error evaluation is investigated and the results of two identification methods are shown in Table 4. The model identification using LASSO has lower number of coefficients than the model identified by ridge regression. In addition, LASSO does not affect the mean square error of the estimated air-fuel ratio. The LASSO uses only the most important features thus simplifying the model automatically. When the model has redundancy, i.e., the measurement data includes noise signal and we attempt to fit polynomial models of various orders, this technique can attenuate the risk of over learning is sue.

TABLE IV. MODEL ERROR EVALUATION

\begin{tabular}{|c|c|c|}
\hline & Ridge regression & LASSO \\
\hline Non-zero coefficient & 7 & 5 \\
\hline Mean error & -0.03 & 0.01 \\
\hline Minimum error & -0.87 & -0.76 \\
\hline Maximum error & 0.47 & 0.54 \\
\hline Squres error & 2.26 & 2.26 \\
\hline Mean square error & 0.15 & 0.15 \\
\hline
\end{tabular}

However, the over learning is not obviously affected in this work because the mean-value data is applied. Therefore, the evident results show only the model simplification. The comparison of these two model performance in real time application is left as future work.

\section{CONCLUSIONS}

The identification results show that the application of LASSO technique can improve the air-fuel ratio model structure compared with identification using ridge regression. This technique can shrink two coefficients to zero at the specified value of the complexity parameter. Additionally, the magnitude of square bias compared with the cause of using ridge regression is not caused by parameter shrinkage. Hence, we can apply the simplified model with five coefficients for air-fuel ratio calculation.

\section{ACKNOWLEDGMENT}

The authors would like to thank Toyota Motor Corporation for their support and fruitful discussions.

\section{REFERENCES}

[1] P. Wibberley, C. Clark. "An investigation of cylinder pressure as feedback for control of internal combustion engines," SAE Technical Paper 890396, 1989.

[2] P. Li, T. Shen, J. Kako, K. Liu. "Cyclic moving average control approach to cylinder pressure and its experimental validation,"Journal of ControlTheory and Applications, Vol. 7(4), pp.345-351, October 2009.

[3] P. Tunestal, J. K. Hedrick. "Cylinder air/fuel ratio estimation using net heat release data," Control Engineering Practice: Advances in Automotive Control, Vol. 11(3), pp.311-318, March 2003.

[4] P. Tunestal. "Estimation of the in-cylinder air/fuel ratio of an internal combustion engine by the use of pressure sensors," Ph.D. Thesis, Lund Institute of Technology, Sweden, 2001.

[5] C. Khajorntraidet and K. Ito. "Simple adaptive air-fuel ratio control of a port injection SI engine with a cylinder pressure sensor,"Journal of ControlTheory and Technology, Vol. 13, No.2, pp.141-150, May 2015.

[6] A. E. Hoerl and R. W. Kennard, "Ridge regression: biased estimationfor nonorthogonal problems," Technometrics Vol.12, pp. 55-67, 1970.

[7] R. Tibshirani. "Regression shrinkage and selection via the Lasso,"Journal of the Royal Statistical Society, Series B,Vol. 58, pp. 267-288, 1996.

[8] T. Hastie, R. Tibshirani and J.Friedman, The elements of ststistical learning: Dat a mining, Inference and Prediction,2nd ed., Springer, New York, 2008.

[9] J. B. Heywood. Internal Combustion Engine Fundamentals. McGrawHill, 1988. 